\title{
How domestic animal genomics can teach human medicine and evolutionary biology
}

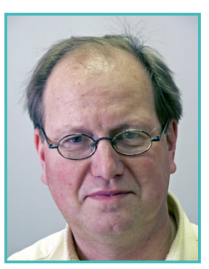

\section{Leif Andersson}

Science for Life Laboratory, Department of Medical Biochemistry and Microbiology, Uppsala University,

Sweden

Domestic animals provide unique opportunities for exploring genotype-phenotype relationships. Firstly, selective breeding during thousands of years has enriched for mutations that have adapted domestic animals to a new environment, i.e. farming under various environmental conditions. Secondly, the population structure is often favorable for genetic studies, large families and more or less closely related subpopulations (breeds). Thirdly, strong positive selection leaves genomic footprints that facilitate positional cloning. The combined use of whole genome resequencing, linkage mapping and linkage disequilibrium (LD) mapping within and between breeds provides a powerful approach for positional identification of both monogenic and multifactorial trait loci. The successful use of this approach for identifying genes underlying phenotypic traits will be illustrated on the basis of our research program in chickens, pigs, dogs, horses and rabbits. Several emerging features as regards the phenotypic evolution of domestic animals will be illustrated including:

- the importance of tissue-specific regulatory mutations:

- the importance of structural changes (duplications, deletions, inversions);

- evolution of alleles at loci under strong directional selection. 